\title{
Correction to: From Grey to Green: Rethinking Setback and MGC Rules as a Sustainable Growth Strategy of Residential Areas - A Case Study of Anannya Residential Area of Chittagong, Bangladesh
}

Rezuana Islam, Kanu Kumar Das, and Samira Binte Bashar

Correction to:

Chapter 6 in: M. Ghosh (ed.), Perception, Design and Ecology of the Built

Environment, Springer Geography,

https://doi.org/10.1007/978-3-030-25879-5_6

The original version of this chapter was published with some citations missing. The revised version has the missed citations added. 